\title{
DESBLOQUEO CONCEPTUAL DE LA CONTROVERSIA SOBRE EL DETERMINISMO DURANTE LA SEGUNDA MITAD DEL SIGLO XIX
}

\author{
Olga VAREla Machado \\ Universitat de València \\ Facultad de filosofía y ciencias de la educación \\ Valencia, España \\ olgavarmac@gmail.com
}

RESUMEN: La física durante la segunda mitad del siglo XIX experimentó una silenciosa pero profunda revisión de sus fundamentos. A pesar de su importancia para los desarrollos posteriores de la física, los estudios históricos y filosóficos sobre dicho periodo son escasos comparados con aquellos de principios del siglo XX. Este trabajo reconstruye algunos aspectos de la física de este periodo usando dos herramientas conceptuales: espacios controversiales de Oscar Nudler y estilo de razonamiento científico de Ian Hacking. El objetivo es mostrar los elementos que produjeron el desbloqueo conceptual de la controversia sobre el determinismo durante la segunda mitad del siglo XIX y principios del siglo XX.

PALABRAS CLAVE: historia de la física, mecánica estadística, estilos de razonamiento científico, espacios controversiales, termodinámica

SUMMARY: During the second half of the nineteenth century, physics go through a noiseless but deep review of its foundations. Despite of the importance for the upcoming developments, the historical and philosophical studies about that time are scarce in comparison to the first decades of the twentieth century. This paper rebuilds some aspects of physics of this time using two conceptual tools: The Controversy Spaces of Oscar Nudler and the Styles of Scientific Reasoning of Ian Hacking. The aim is showing the elements that led to the controversy about determinism during the second half of the nineteenth century and beginning of the twentieth.

KEY WORDS: history of physics, statistics mechanics, styles of scientific reasoning, controversy spaces, thermodynamics

\section{Introducción}

El propósito de este estudio es analizar algunos de los elementos que propiciaron el desbloqueo conceptual de la controversia sobre el determinismo. Dicho desbloqueo culmina durante la primera mitad del siglo XX con la consolidación de la mecánica cuántica. Mi interés se enfoca en presentar los aspectos que consideramos más relevantes y que, iniciándose ya en el siglo XVIII, durante la segunda mitad del 
siglo XIX configuraron las condiciones necesarias para que se desarrollara la discusión sobre el determinismo al interior de la teoría cuántica. La importancia de este episodio en la historia de la ciencia no ha tenido la vistosidad que sí han tenido los eventos acaecidos durante la primera mitad del siglo XX (con el advenimiento de la teoría de la relatividad y la mecánica cuántica) y que han ocupado el interés central, tanto de historiadores como de filósofos de la ciencia. Sin embargo, esta revolución del siglo XX no habría sido posible sin las condiciones y elementos conceptuales que durante el siglo XIX entraron en el escenario de la física. Con el fin de iluminar y resaltar la importancia de esos acontecimientos, este estudio se centra en un periodo que hemos llamado precontroversial respecto del determinismo. Aquí presentaré los elementos conceptuales más relevantes para que la controversia sobre el determinismo se desarrolle.

Partimos, además, de una concepción de la ciencia donde el desacuerdo es parte estructural de su dinámica de desarrollo. Y consideramos que la comprensión de estos desacuerdos, el modo en que evolucionan en el tiempo y el papel epistemológico que desempeñan en la construcción de la ciencia nos aportará material relevante para el análisis, tanto filosófico como histórico. Con este propósito, el trabajo adopta un marco teórico basado en dos herramientas conceptuales: por un lado, el modelo de espacios controversiales (Controversy Spaces) de Oscar Nudler $(2002,2004,2009)$ y, por otro, la noción de estilo de razonamiento científico (Style of Scientific Reasoning) propuesta por Ian Hacking $(1982,1992 a)$.

El modelo de espacios controversiales resulta útil para esta reconstrucción porque permite enmarcar conjuntos de controversias interrelacionadas en largos periodos de tiempo, posibilitando un tipo de visión big picture. Esta interrelación facilita la comprensión de la forma en que las distintas discusiones y desacuerdos se fueron refinando y moldeando, así como los conceptos y los programas de investigación relacionados. Por su parte, el concepto de "estilo de razonamiento científico" nos permite analizar los acuerdos previos de la comunidad científica, así como la forma en que dichos acuerdos van cambiando en la medida en que se instala un nuevo estilo. Esto resulta fundamental en el desarrollo de algunas controversias, pues aporta nuevos elementos para comprender tanto la discusión como el posterior acuerdo. Para este caso de estudio en particular, nos interesa analizar cómo la introducción del estilo de razonamiento estadístico propició el desbloqueo conceptual de la controversia sobre el determinismo. Por eso, tanto el modelo de Nudler, como el concepto de Hacking se asocian aquí como herramientas de análisis 
para este caso, permitiéndonos construir una visión amplia en cuanto a la historia conceptual del determinismo y su relación con otras controversias.

Sobre esta base, el presente artículo se estructura partiendo de un marco teórico (sección 2), en el que presentaré a grandes rasgos el modelo de espacios controversiales y el concepto de estilos de razonamiento, así como la forma en que se articulan en el presente trabajo. Luego, expondré algunas aclaraciones sobre el núcleo de la controversia, es decir, el problema del determinismo (sección 3). Allí presentaré algunas clarificaciones sobre el término que serán útiles para el análisis de este episodio histórico. A continuación (sección 4) brindaré una breve descripción de la discusión sobre el determinismo entre los siglos XVII y XVIII, lo cual permitirá comprender el bloqueo conceptual que estanca la controversia durante el siglo XIX (sección 5). Posteriormente, señalaré el papel fundamental que desempeñaron la Segunda Ley de la Termodinámica y las discusiones acerca de su interpretación estadística en el establecimiento de las bases necesarias para el desbloqueo conceptual de la controversia (sección 6). Finalmente, concluiré esta reconstrucción histórico-conceptual indicando dos nuevos elementos de acuerdo que se introducen en la física durante la primera década del siglo XX (sección 7): la presentación canónica de Gibbs de la mecánica estadística y la aceptación de la hipótesis atómica como una forma adecuada de la estructura de la materia.

2. Marco teórico: espacios controversiales y estilos de razonamiento

\subsection{Espacios controversiales}

En sus trabajos de la década de 2000, Oscar Nudler centró su interés en el estudio de las controversias desde un punto de vista filosófico. Desde esta perspectiva propuso un modelo de análisis que permite analizar las controversias durante periodos más prolongados que los identificados tradicionalmente por los historiadores. Su modelo permite trascender las discusiones puntuales entre actores concretos y proporciona una estructura capaz de brindar una aproximación filosófica que facilite el análisis de elementos epistemológicos y conceptuales. Las ventajas que presenta frente a los análisis de controversias científicas puntuales es que reconcilia la continuidad y la discontinuidad histórica, muestra factores que se conservan y factores que se descartan, facilitando una visión más panorámica, fundamental para el análisis filosófico. 
El estudio de los espacios controversiales se aborda desde dos perspectivas: la estructural y la dinámica. La perspectiva estructural se refiere a los elementos conceptuales, ideológicos, sociales y culturales que caracterizan el espacio controversial y los diferentes debates asociados (la red de controversias). La estructura se caracteriza al identificar los siguientes elementos: (i) una red de controversias interrelacionadas constituidas por diferentes temas polémicos que, o bien se discuten simultáneamente, o bien aparecen y desaparecen a lo largo del periodo en el que se desarrolla el espacio; (ii) un foco, conformado por el conjunto de cuestiones problemáticas que se evidencian de forma explícita en un espacio controversial, es decir, los temas y problemas que ocupan la atención central de los actores de las controversias; (iii) un terreno común, que se refiere a los supuestos compartidos por los actores en el marco de la controversia, pues es necesario un mínimo acuerdo entre los actores para que la discusión tenga sentido; y (iv) un núcleo, concepto que Lombardi (2009) introdujo y que pretende asegurar un criterio de identidad para el espacio controversial a través de los cambios que experimenta durante el tiempo de su vigencia.

Puesto que los espacios controversiales no son estáticos, sus temas de discusión cambian en la medida en que los problemas o las preguntas se van resolviendo o abandonando. Las controversias se nutren y evolucionan durante el tiempo en el que interaccionan dentro de la red de controversias. El modelo de Nudler facilita observar la dinámica del espacio controversial durante su periodo de vigencia a través de los siguientes elementos. (i) Bloqueo conceptual: periodo durante el cual una controversia se estanca y los diferentes actores implicados pierden interés en los problemas que se estaban discutiendo o consideran que se han resuelto. Los motivos de un bloqueo conceptual podrían identificarse con las categorías de clausura, resolución o abandono de controversias que autores como McMullin (1987), Beauchamp (1987) o Mazur (1981) formulan. La diferencia entre las propuestas de estos autores y el caso de los espacios controversiales es que, por observar periodos más largos, en este último caso, el foco del espacio o los problemas asociados a él pueden reaparecer por diferentes circunstancias. Por lo tanto, un bloqueo conceptual no se considera definitivo, sino que se concibe como parte del movimiento natural de la controversia. (ii) Desbloqueo conceptual: así como los actores de una controversia pueden perder interés en ella, asimismo otros actores, o ellos mismos, pueden retomar el interés debido a la aparición de nuevos métodos, nuevas preguntas, nuevas herramientas conceptuales, entre otros factores que les permitirán profundizar la 
discusión o retomarla desde otras perspectivas. Éste es uno de los elementos centrales en el presente estudio, pues lo que nos interesa poner en evidencia aquí son los elementos que contribuyeron al desbloqueo conceptual de la controversia sobre el determinismo durante la segunda mitad del siglo XIX hasta la primera década del siglo XX. El último elemento de la dinámica es (iii) la refocalización, que se refiere a los cambios que sufre el foco de un espacio controversial. Este proceso es uno de los más interesantes para el análisis de los espacios controversiales, pero en el presente caso de interés la refocalización se desarrolla en un periodo fuera de los límites de este estudio. El análisis de este episodio lo presentaré en un trabajo futuro.

\subsection{Estilos de razonamiento}

El concepto de estilo de razonamiento es el hilo conductor que nos permitirá analizar el cambio conceptual a lo largo del devenir del espacio controversial en estudio. Ian Hacking se inspira en la propuesta historiográfica de Crombie (1995), quien propone seis categorías, a las que denomina estilos de pensamiento científico europeo, para caracterizar la forma en que se ha pensado y validado la ciencia en Occidente. Si bien Hacking parte de estos seis estilos de pensamiento, avanza un paso más: con ellos pretende encontrar una solución adecuada al problema de la objetividad en las ciencias:

Mis estilos de razonamiento [...] son parte de lo que necesitamos para entender aquello que llamamos objetividad. No porque los estilos sean objetivos (es decir, que hayamos encontrado la mejor forma de llegar a la verdad), sino porque ellos han fijado lo que es ser objetivo (las verdades de cierto tipo son solo lo que obtenemos al realizar cierto tipo de investigaciones, respondiendo a ciertos estándares). (1992a, p. 4; la traducción es mía.)

La pregunta por la objetividad es determinante en el análisis de las controversias, pues son episodios en los que no existe un consenso dentro de la comunidad científica: el criterio de objetividad sobre los temas en disputa también está sujeto a discusión. Por este motivo, el concepto de estilo de razonamiento que Hacking propone sirve para comprender cómo cambia la objetividad en la ciencia, posibilitando acuerdos sobre temas en los que previamente no fue posible conciliar.

Hacking resalta también el problema de la verdad o falsedad de los enunciados científicos. Las controversias giran precisamente en torno a este problema: cuando se presenta una nueva solución a una pregunta científica, dicha solución puede cuestionarse con respecto a su 
valor de verdad, o los métodos que se utilizan para respaldarla pueden no ser considerados adecuados para acercarse a resultados verdaderos. Lo importante para el enfoque de Hacking no es el veredicto acerca de la verdad o falsedad de un enunciado científico, sino cómo ciertos enunciados tienen la posibilidad de ser juzgados como verdaderos o falsos por la comunidad científica: "que una proposición comience a jugar como candidata o no a ser verdadera o falsa depende de si tenemos modos de razonar sobre ella" (1982, p. 49; la traducción es mía). En este sentido, la noción de estilo de razonamiento es una herramienta relevante para analizar diferentes tipos de controversias, pues permite identificar aspectos determinantes para su análisis, tanto epistemológico como histórico.

A los seis estilos de pensamiento científico que Crombie presenta, Hacking agrega dos más. Los ocho estilos de razonamiento vigentes en las ciencias occidentales son, según el autor: (i) la postulación de las ciencias matemáticas; (ii) el desarrollo del experimento a través de la observación y la medición; (iii) la construcción hipotética de modelos por analogía; (iv) el ordenamiento de la variedad mediante la comparación y la taxonomía; (v) el análisis estadístico de regularidades en poblaciones y el cálculo de probabilidades, y (vi) la derivación histórica del desarrollo genético (evolución). Hasta aquí los estilos de pensamiento que Crombie propone, más los dos estilos agregados por Hacking: (vii) el estilo de laboratorio, y (viii) el estilo algorítmico. En esta reconstrucción mostraremos cómo el quinto estilo: el análisis estadístico de regularidades en poblaciones y el cálculo de probabilidades, al ser introducido en la física, se convierte en el motor de transformación del espacio controversial, desencadenando preguntas profundas acerca del significado de las leyes físicas, del determinismo y de la realidad.

\section{El núcleo del espacio controversial: el concepto de determinismo}

A la hora de reconstruir este espacio controversial se presentan dos dificultades asociadas con el núcleo de este espacio. La primera de ellas se debe a que el problema del determinismo tiene una larga tradición discursiva en la filosofía y, por tanto, es necesario delimitar los alcances temporales del debate. La controversia acerca del determinismo se extiende a lo largo de la historia de Occidente como la filosofía misma: aparece ya en las críticas de Aristóteles al determinismo de los atomistas y establece una diferencia sustancial entre el estoicismo (determinista) y el epicureísmo (indeterminista) durante el periodo helenista. En el medioevo cristiano la discusión aparece en 
la contraposición entre el determinismo frente a la omnipotencia de Dios, pues la predestinación es una consecuencia lógica y necesaria de la omnisciencia divina. Por esta razón, y por la forma en que la controversia ha mutado y se ha adaptado a diferentes épocas y problemas, el determinismo llega a la modernidad como un término con un amplio rango de aplicación. Se lo ha relacionado filosóficamente con otros conceptos como el de causalidad, necesidad, ley natural o incluso con la adecuación de las matemáticas al mundo. Algunos autores, como Earman (1986), por ejemplo, caracterizan esta discusión como una Torre de Babel, es decir, más como un problema de lenguaje y de definición que como un problema ontológico.

La segunda dificultad, no menos importante, es que el problema del determinismo se discutió en el contexto de diferentes teorías físicas, es decir, para diferentes conjuntos de fenómenos físicos; en cada una de las teorías las implicaciones de la adopción o no del determinismo son diferentes y se relacionan con distintos puntos de vista epistemológicos, metodológicos y ontológicos. Por este motivo, proponer una noción prescriptiva de determinismo implicaría cancelar muchos de los matices de la controversia y, sobre todo, no permitiría ver cómo la noción evolucionó a través del debate. Sin embargo, es importante delimitar algunos aspectos y plantear algunos de los matices más relevantes del uso del término "determinismo" en la tradición occidental durante el periodo que nos ocupa, con el fin de contar con algunos puntos firmes para el análisis del desbloqueo conceptual de la controversia.

A continuación, presentaré algunos significados usuales del concepto de determinismo en la tradición científica entre el siglo XVIII y principios del siglo XX, así como los diferentes matices de la aplicación del término, con miras a comprender mejor el núcleo del espacio controversial. Y dado que el problema del determinismo en la física ha sido abundantemente estudiado en las últimas décadas, es posible encontrar buenos tratados que analizan el problema conceptual a profundidad. Aquí solo presentaremos un breve esbozo, necesario para que la discusión posterior resulte inteligible.

\subsection{Formas del determinismo}

En general, el determinismo ha sido concebido en física en dos sentidos: por un lado, como característica o propiedad de un objeto o fenómeno; por otro lado, como conexión constante y unívoca de eventos. En la primera acepción "determinado es aquello que tiene propiedades definidas $y$, por tanto, puede ser caracterizado de 
un modo inequívoco." (Lombardi 2000, p. 19). En cambio, como conexión constante y unívoca, el término "determinación" implica la relación entre eventos tanto reales como ideales en una línea de tiempo bien definida. En este sentido, el determinismo supone la existencia de líneas unívocas de sucesión temporal para todos los sucesos que conforman la historia del universo. Esta definición es la que se relaciona con problemas referidos a las nociones de realidad, causalidad, necesidad y azar.

Asimismo, dependiendo del tipo de ítems a las que se aplique el determinismo, éste puede adoptar un sentido semántico, gnoseológico (epistemológico) u ontológico. (Lombardi 2000, p. 20). El determinismo semántico evalúa el formalismo de las teorías. Se decide si una teoría es determinista o no analizando si sus leyes, representadas por ecuaciones diferenciales, cumplen con ciertas características: el formalismo deberá garantizar la conexión constante y unívoca de los eventos a través del tiempo. Algunos autores actuales, sobre la base de este criterio, han reconsiderado la idea tradicional de que la mecánica clásica es determinista y la mecánica cuántica es indeterminista. ${ }^{1}$

Por otra parte, el determinismo epistémico se refiere a la posibilidad de conocer los estados de los sistemas físicos en cuestión. Incluso en el caso en que la teoría presente un formalismo que se adecue a la definición de determinismo en sentido semántico, lo que interesa aquí es discutir si es humanamente posible conocer, por ejemplo, con suficiente precisión las condiciones iniciales del estado de un sistema con el fin de que puedan conocerse sus estados futuros dentro de una precisión acotada. Esto significa que el énfasis no se encuentra en el lenguaje sino en la capacidad humana de conocer la evolución del sistema a través del tiempo.

Finalmente, el determinismo ontológico apunta a una condición más fundamental: pretende garantizar las condiciones del determinismo en la realidad misma. Si bien se ha evitado este enfoque ontológico del problema en la tradición filosófica analítica del siglo XX, algunos autores consideran que, en último término, toda discusión sobre el determinismo tiene como telón de fondo un enfoque realista: ¿es la naturaleza de los fenómenos físicos determinista o no? Es decir, la pregunta por el determinismo, aunque se formule con énfasis en los aspectos semánticos o epistémicos, en última instancia es siempre una pregunta ontológica. En este sentido, Lombardi afirma:

\footnotetext{
${ }^{1}$ Véase, por ejemplo, Earman 1986; Butterfield 1998; Lombardi 2000; Bishop 2006.
} 
Si se reflexiona acerca de estas definiciones provisionales a la luz de la historia de la filosofía, puede comprobarse que, salvo en épocas recientes, el problema del determinismo se formuló en términos metafísicos: cuando James (1897) se refería a un futuro sin posibilidades ocultas en su seno no aludía a cuestiones metodológicas o gnoseológicas, sino a las características de la realidad misma. En otras palabras, históricamente el núcleo central de la noción de determinismo apunta a una idea referida al plano ontológico. (2000, p. 21)

Earman también concuerda con este punto de vista cuando descarta el problema lingüístico del determinismo (1986, pp. 20-21). Adicionalmente, las relaciones entre determinismo, causalidad, necesidad y ley natural también apuntan en esta dirección ontológica, como se verá a continuación.

\subsection{Determinismo, causalidad y predictibilidad}

Estos diferentes sentidos de la noción de determinismo aparecen fuertemente asociados con las nociones de causalidad, predictibilidad y ley natural. En la tradición filosófica estas asociaciones suelen considerarse resultado de errores conceptuales o de una inadecuada elucidación de los términos involucrados. Aquí, por el contrario, resaltaremos este tipo de asociaciones como parte importante de la huella histórica de la evolución de dichos conceptos. Un esbozo general muy ilustrativo de esta evolución y de las razones que enlazaron estos conceptos puede encontrarse en Crombie 1995.

En términos generales, se asocia determinismo con predictibilidad cuando lo que está en juego en la discusión apunta al sentido semántico o epistémico de la noción de determinismo. Por otro lado, causalidad, determinismo y ley física aparecen asociados cuando el énfasis de la discusión es ontológico, aunque pueden observarse algunos casos en los que el interés también es epistémico. Puede afirmarse, entonces, que la discusión acerca del determinismo tiene un parentesco cercano con la noción de predictibilidad en ciertos autores y en contextos de controversias ligados al sentido semántico y epistémico del término, mientras que se conecta con la noción de causalidad cuando el interés de las discusiones se remite al plano ontológico o metafísico, por lo que entran en juego los problemas sobre el realismo, la necesidad y la ley natural. Sin embargo, no se puede trazar una clara línea divisoria entre estos conceptos hasta la primera mitad del siglo XX, como veremos más adelante. ${ }^{2}$

${ }^{2}$ Véase, por ejemplo, Forman 1984 [1971], pp. 102-110. 
Con el término "causalidad" sucede algo semejante, ha sido utilizado en la historia de la filosofía en muchos sentidos que pueden asociarse de forma más fuerte o más débil con el determinismo. Por ejemplo: causalidad como equivalente a las leyes de la mecánica clásica, o a la conservación de la energía y el momento, a la visualización en el espacio y el tiempo, a la ausencia de acción a distancia, a la acción por contacto, o a la descripción mediante ecuaciones diferenciales. Estas asociaciones pueden agruparse también en semánticas, epistémicas u ontológicas, y aparecen tanto en los defensores de un enfoque causal, como en los que pretenden negar su validez:

En cada caso, sin embargo, estas definiciones especiales de causalidad, y a fortiori, el requisito general de determinación sin ambigüedad, fueron considerados como equivalentes a la suposición de la comprensibilidad de la naturaleza, y repudiados o defendidos como tales. (Forman 1984 [1971], p. 109)

En el periodo comprendido entre los siglos XVIII y XIX, el concepto de determinismo no fue objeto del análisis con el que se lo aborda en la actualidad, debido en parte a que físicos y filósofos de este periodo no discutían las implicaciones de una visión determinista en términos ontológicos, sino que la daban por sentado. Esta ausencia de confrontaciones respecto del determinismo se evidencia precisamente en la ambigüedad e imprecisión del término. Fue solo a partir de la introducción de discusiones provenientes de ámbitos ajenos a la física que la noción adquirió su actual significación. Así, en la medida en que los métodos estadísticos en la física empezaron a aceptarse tuvo sentido discutir sus implicaciones en la noción de ley natural y su relación con el concepto modal de necesidad, a la vez que se fue modificando y restringiendo el significado del determinismo para las teorías físicas.

\section{La controversia sobre el determinismo entre los siglos XVII y XVIII}

En la modernidad la discusión central sobre el determinismo se centró en compatibilizar el libre albedrío humano en un mundo mecanicista determinado por leyes universales. Esta fue una discusión recurrente entre muchos pensadores de la modernidad (Descartes, Spinoza, Leibniz, Kant). El centro de la discusión aquí no fue el determinismo ontológico, sino cómo definir la libertad humana de modo que fuera coherente con dicho determinismo ya presupuesto. La introducción de la mecánica y de la imagen del mundo-reloj agudizó en 
un primer momento estas discusiones: el problema era compatibilizar el movimiento de la materia (determinista) con el comportamiento humano. Algunos autores se decantaron por un determinismo intrínseco del comportamiento humano, dado que este último es también movimiento de materia. Otros intentaron preservar la libertad moral humana más allá del mecanismo de relojería que constituye el mundo.

Fueron precisamente estas discusiones sobre el libre albedrío, en contraste con el mecanicismo, las que llevaron a diversos pensadores del siglo XVIII a plantear una definición de determinismo que habría de vincularlo con la doctrina de la necesidad de forma más radical de lo que ya había sido insinuada por los primeros pensadores modernos. Esta vinculación se vio reforzada a partir de la aplicación de los métodos estadísticos al análisis de poblaciones, en lo que Hacking (1992b) ha llamado la avalancha de números impresos, que se dio durante las primeras décadas del siglo XIX. Durante este periodo muchos autores creyeron ver un verdadero determinismo subyacente en la conducta humana: casos como el suicidio, la criminalidad, e incluso, la enfermedad, parecían ser solo consecuencia de la ley de los grandes números. Es así como se establece una vinculación explícita entre determinismo y causalidad que profundiza el sentido ontológico del término "determinismo".

Adicionalmente, y en estrecha relación con la convicción de un determinismo ontológico, se impone la confianza en la primacía de la razón para el conocimiento del mundo y el consecuente rechazo del azar intrínseco. Estos dos aspectos están íntimamente ligados con una visión ontológicamente determinista y pueden relacionarse fácilmente también con la visión mecanicista de la naturaleza. Autores como De Moivre se refieren al azar de un modo despectivo, convencidos de la existencia de una explicación racional detrás de todos los fenómenos:

El azar, en los escritos o discursos ateos, es un sonido absolutamente insignificante: no aporta determinación a ningún modo de existencia; ni siquiera a la existencia misma; no puede definirse ni entenderse, ni tampoco puede afirmarse ni negarse ninguna proposición que le concierna, excepto ésta: "el azar es una mera palabra". (1738, p. 241; la traducción es mía.)

También Hume se expresa en términos similares: "Está comúnmente admitido por los filósofos que lo que el vulgo llama azar no es sino una secreta y oculta causa" (1888 [1739], p. 130); "Está universalmente admitido que nada existe sin una causa de su existencia y que el azar, cuando se lo examina estrictamente, es una mera palabra 
negativa y no significa ninguna fuerza real que pueda tener un ser en alguna parte de la naturaleza" (1902 [1748], p. 95).

El argumento de De Moivre descarta la existencia del azar sobre la base de su supuesta incomprensibilidad. Dado que el azar no puede abordarse a través de la razón, entonces no puede considerarse como parte constituyente de la naturaleza. Aquí se manifiesta una asociación fuerte entre ley, determinación y racionalidad. Hume en cambio, resalta la necesidad o causalidad subyacente como argumento para la negación del azar. Es decir, solo se habla de azar cuando se carece de un conocimiento completo de las causas, sin que esto signifique que la naturaleza no las contenga en sí misma. También aquí la relación entre determinismo, racionalidad e inteligibilidad es directa. De acuerdo con Hacking, estas afirmaciones de Hume sembraron la duda respecto del determinismo epistémico:

¿Por qué cada una de las citas [de Hume] comienza con: "está comúnmente admitido", "está universalmente admitido", "es universalmente reconocido"? ¿Ponen estas frases la carga de la prueba en otros filósofos en lugar de expresar el acuerdo de Hume? Pero aquello de que dudaba Hume era algo diferente; no dudaba de la realidad de la necesidad, sino que dudaba de nuestro conocimiento de ella. Únicamente se mofaba de las pretensiones de conocer las operaciones íntimas de la naturaleza. (1990, p. 34)

Lo que aquí señala Hacking es un escepticismo respecto del determinismo en su sentido epistémico.

Desde esta perspectiva, en el ámbito de la física el caso de Laplace es paradigmático. Su famoso Essai Philosophique sur les Probabilités comienza explicando la probabilidad del siguiente modo:

Todos los acontecimientos, incluso aquellos que por su insignificancia parecen no atenerse a las grandes leyes de la naturaleza, no son sino una consecuencia tan necesaria como las revoluciones del sol. Al ignorar los lazos que los unen al sistema total del universo, se los ha hecho depender de causas finales o del azar, según que ocurrieran o se sucedieran con regularidad o sin orden aparente, pero estas causas imaginarias han ido siendo descartadas a medida que se han ido ampliando las fronteras de nuestro conocimiento, y desaparecen por completo ante la sana filosofía que no ve en ellas más que la expresión de nuestra ignorancia de las verdaderas causas. (1996 [1814], p. 24)

Este punto de vista en la física puede considerarse como parte del terreno común del espacio controversial de la época, y refleja precisamente el estado de bloqueo conceptual respecto al determinismo 
ontológico. Todo fenómeno que se aleje de la determinación precisa esconde información que no hemos podido determinar a través de nuestras observaciones. Pero no podemos dudar de que esa información existe efectivamente en el mundo.

\section{El bloqueo conceptual de la controversia durante el siglo XIX}

Las relaciones entre el determinismo, la doctrina mecanicista y paulatinamente la asociación con las ideas de necesidad, tal como se acaban de exponer, conducen al bloqueo conceptual de esta controversia. Esto significa que las discusiones se agotan sobre la base de un acuerdo generalizado acerca del determinismo de las leyes físicas newtonianas. Esto no implica que no existieran debates respecto de estas leyes, sino que el carácter determinista de tales leyes no estaba en discusión. En términos generales puede afirmarse que los físicos de finales del siglo XVII adoptaron de un modo irrestricto las leyes de Newton, así como sus implicaciones metafísicas: el significado del concepto de ley física, las posibilidades de predicción a partir de estas leyes, las respectivas consecuencias epistemológicas y de racionalización de la naturaleza y, por consiguiente, los límites del conocimiento humano acerca del mundo. Éste es un primer aspecto del terreno común que puede relacionarse con un consenso general acerca del determinismo ontológico.

Durante este periodo, otro de los acuerdos generalizados que forman parte del terreno común del espacio controversial acerca del determinismo es el correspondiente a los métodos y valores de la mecánica clásica. Si acordamos con Crombie y Hacking en los estilos de pensamiento enumerados anteriormente, es posible postular los siguientes elementos compartidos por todos los actores de este espacio: (i) la metodología propia del pensamiento matemático, (ii) la relevancia de la observación y la medición, y (iii) la construcción de modelos por analogía. Estos tres estilos sientan las bases metodológicas y metafísicas para la física de los siglos XVIII y XIX. El principal programa de investigación a lo largo de esta época fue formular las nuevas teorías de acuerdo con el punto de vista mecánico de la naturaleza, cuyo supuesto central era el movimiento de la materia como base de todos los fenómenos físicos, y cuyo programa de reducción pretendía explicar todo lo físicamente real a partir de dicho movimiento y del conocimiento de sus causas.

Este programa de reducción habría de extenderse hasta los primeros años del siglo XX. Por ejemplo, aún en fechas tan tardías como 1899, Heinrich Hertz escribía: "Todos los físicos estamos de acuerdo 
en que el problema de la física consiste en rastrear los fenómenos de la naturaleza hasta las leyes simples de la mecánica" (1899, p. xxii; la traducción es mía). Sin embargo, Klein (1973) afirma que, para la época en la que Hertz escribe estas palabras, ya no todos los físicos estaban de acuerdo con que la meta de la física fuera explicar el mundo en términos mecánicos. Algunas corrientes alternativas, como el energetismo, se ubicaron en el centro del debate poniendo en duda los principios del mecanicismo. El planteamiento central del movimiento energetista apuntaba a la unificación de todos los fenómenos de la física en términos de energía; si bien muchos energetistas no eran mecanicistas, compartían con estos las aspiraciones reduccionistas para la física. Algunos de los defensores del energetismo fueron abiertamente anti-newtonianos; otros, sobre todo los ingleses, fueron mecanicistas.

La idea era que se podrían reducir las leyes de Newton, y sus derivados, a los principios y leyes más generales de la energía sin necesidad de recurrir a las que Mach llamó "ficciones convenientes" de los átomos y las moléculas. Este movimiento no se restringió solo a Alemania, sino que tuvo influencias en Francia, Suecia, Italia y Estados Unidos y no se limitó solo a la física, sino que también intentó ser un marco conceptual desde el cual entender fenómenos humanos como la voluntad y la felicidad, en consonancia con un espíritu de época anti-materialista. (Alvarez 2019, p. 93)

Así pues, a pesar de que durante el siglo XIX podemos encontrar un programa común entre los físicos y la sensación general de acuerdo y estabilidad, una mirada más detallada de finales de siglo muestra cómo emergió un foco rico en discusiones de todo tipo:

Las dos nuevas ciencias de la termodinámica y la electrodinámica, ambas definidas desde mediados de siglo, diferían en muchos aspectos de la mecánica clásica y, sin embargo, estaba muy extendida la creencia de que se podían entender de forma mecánica, de hecho, tal comprensión ya había sido lograda. James Clerk Maxwell no vio contradicción entre su teoría de campo del electromagnetismo y la mecánica de Newton. (Kragh 2014, p. 3; la traducción es mía.)

Es precisamente en la segunda mitad del siglo XIX que el espacio controversial comienza a adquirir movimiento, ve entrar y salir algunos temas del campo visible de las discusiones.

El terreno común aceptado por el grueso de la comunidad científica incluyó, en este periodo, el valor de la demostración empírica, la 
importancia de las matemáticas y del aparato formal para la descripción del mundo y el criterio de realidad para evaluar la adecuación de las teorías al mundo físico. Al respecto afirma Kragh:

La mecánica newtoniana, o más bien las versiones más avanzadas de la mecánica con raíces en las leyes de Newton, sin duda, se tenía en gran estima a fines del siglo XIX. Se aceptaba ampliamente que el objetivo de la física, o incluso su definición, era la reducción de todos los fenómenos físicos a los principios de la mecánica. (2014, p. 3; la traducción es mía.)

Con este breve panorama no se pretende repetir la idea de que la física durante el siglo XIX fuese aburrida; solo se intenta resaltar la existencia de grandes acuerdos que permitieron una cierta cohesión en la comunidad científica y de un evidente fortalecimiento de la visión mecanicista del mundo que estos acuerdos respaldaban.

6. El desbloqueo conceptual de la controversia: la irrupción de la estadística a finales del siglo XIX

El determinista afirma que el mundo opera mediante leyes de la naturaleza fijas y causales. El indeterminista replica que esas leyes solo pueden ser la consecuencia de la ley de los grandes números aplicada a un gran número de hechos (Hacking 1990, p. 156)

Como veremos a continuación, la separación de las nociones de determinismo, causalidad, necesidad y ley física que se produjo a principios del siglo XX necesitó la confluencia de diferentes factores, entre ellos la introducción del estilo de razonamiento estadístico (Hacking 1975, 1990), la interpretación de la segunda ley de la termodinámica en términos de probabilidad (Brush 1976, 1983, 2003) y la búsqueda de una comprensión más profunda de la irreversibilidad (Lombardi 2009). En esta etapa de la controversia nos centraremos en el papel que desempeña el estilo estadístico al impulsar el renacimiento de la controversia acerca del determinismo como resultado de su desbloqueo conceptual. Como señala Lombardi en su estudio sobre el determinismo: "Según el historiador de la ciencia Stephen Brush (1976), la confianza en el determinismo comienza a mermar ya en la segunda mitad del siglo XIX, con la irrupción de los métodos estadísticos en la física a través de la Teoría Cinética de los Gases" (2000, p. 13). La 
intrusión de la idea de ley estadística, con la que muchos pensadores comenzaban a reemplazar el modelo ideal de ley mecánica, favoreció la discusión acerca del sentido físico de este nuevo tipo de ley. La red de controversias en la que el problema del determinismo habría de insertarse ya había instalado ciertos temas de debate: la larga discusión sobre el libre albedrío, como vimos en un apartado anterior; las disputas sobre la estructura continua o discontinua de la materia (a partir de la reintroducción de la hipótesis atomista) y la controversia sobre la naturaleza de la luz también estaban vigentes en esta época; además, en la segunda mitad del siglo XIX la controversia con los energetistas entra en escena, y más o menos en el mismo periodo la controversia sobre el carácter estadístico de la termodinámica.

Es muy importante resaltar cómo la introducción de los métodos estadísticos en la física, acompañada de la hipótesis atomista, genera un movimiento desde el terreno común hacia el foco de este espacio controversial, sacando a la luz, en diferentes momentos, las discusiones sobre el determinismo, la probabilidad y la irreversibilidad. Durante la primera mitad del siglo XIX el uso de la estadística en otras disciplinas, como la medicina, la fisiología y la biología, ya había instalado la discusión acerca de las regularidades estadísticas observadas en colecciones de datos cada vez más grandes y de las probabilidades asociadas a ellas (la controversia alrededor de los métodos estadísticos para el análisis de poblaciones se presenta en detalle en Hacking 1990). Los matemáticos y los estadígrafos desarrollaban métodos cada vez más refinados para analizar dichas regularidades, con lo que surge la pregunta acerca de su significado conceptual: ante un conjunto enorme de datos referentes a un mismo fenómeno, algunos datos son extremos (máximos y mínimos), pero la mayoría de ellos se agrupan alrededor de ciertos valores; la pregunta que se impone aquí es ¿qué significan esas regularidades? Y ¿cómo puede interpretarse la probabilidad en el marco de una visión mecanicista del mundo? Estas preguntas irán guiando la aparición del desacuerdo sobre el determinismo ligado a una discusión más sutil acerca de la pertinencia de las relaciones causales y su relación con las probabilidades.

Es en este periodo, a fines del siglo XIX, que se crean "nuevos objetos", propios del estilo de razonamiento estadístico, y se avanza hacia la autonomía de las leyes estadísticas. En ese sentido, una consecuencia de intentar reducir la segunda ley de la termodinámica a las leyes de la mecánica clásica es la aparición de estos nuevos objetos para la física: la concepción de un gas como un agregado de partículas en conjuntos propios que Boltzmann llama ergode en 1884 y que 
más tarde Gibbs modifica en términos de ensemble. Estos objetos tendrán las características propias de un objeto estadístico, que solo puede ser conocido y estudiado a través del estilo de razonamiento estadístico, tal y como fueron estudiadas las poblaciones por Quetelet en 1844. Adicionalmente, la autentificación de las leyes estadísticas se produce durante este periodo, alargándose para la física hasta la primera década del siglo XX. Se verá cómo estos elementos están relacionados con el núcleo de este espacio controversial.

\section{1. Interpretación estadística de la Segunda Ley \\ de la termodinámica}

La historia de la termodinámica durante la segunda mitad del siglo XIX es clave para delimitar al menos cuatro controversias importantes, todas ellas relacionadas con el determinismo. En primer lugar, aparecen las discusiones acerca de la reducción de los principios de la termodinámica a la mecánica; esta controversia se enmarca en las diferentes disputas alrededor del reduccionismo e incluye la discusión con los energetistas. En segundo lugar, surge la discusión sobre la irreversibilidad, precisamente como consecuencia de esos intentos de reducción de la termodinámica a la mecánica. Finalmente, reaparece la discusión sobre la constitución de la materia debido a la introducción de la hipótesis atómica en la Teoría Cinética de Gases. Cada una de estas controversias amerita un estudio detallado; aquí, por cómo hemos delimitado el enfoque de este estudio, nos centraremos en mostrar cómo encajan las piezas para el posterior desbloqueo conceptual de la controversia sobre el determinismo. Veremos cómo la introducción de las discusiones sobre probabilidad e irreversibilidad, a través de los intentos de reducción de la Segunda Ley a la mecánica, establecen los elementos necesarios para el posterior desbloqueo.

La primera ley estadística que aparece en la física es la ley de distribución de velocidades, formulada en 1861 por Maxwell. Con esta ley se introducen los métodos estadísticos para el cálculo de las velocidades de las moléculas de un gas en estado de equilibrio. "El núcleo de su aporte consiste en haber logrado definir el estado macroscópico de equilibrio termodinámico en términos mecánicos microscópicos" (Lombardi 2009, p. 132). Sin embargo, la aparición de esta ley estadística no fue suficiente para lograr su autonomía en los términos que propone Hacking, pues se requerían nuevas explicaciones que autentificaran el estilo estadístico: como ya hemos mencionado, fue necesario presentar nuevos objetos, propios del estilo. Los primeros pasos para la autentificación del estilo incluyen, 
además de la postulación de la ley de distribución de Maxwell como primera ley estadística, la introducción de la noción de entropía por Clausius en 1865, y las propuestas de Bolztmann, quien entre 1866 y 1877 publicó una serie de trabajos que lo llevarían a plantear finalmente la ley de distribución de Maxwell-Boltzmann (un refinamiento de la anterior ley de distribución de Maxwell). La consecuencia más importante de esta nueva versión de la ley de distribución fue la primera interpretación puramente probabilística de la Segunda Ley, en la que las explicaciones causales fueron sustituidas por explicaciones estadísticas, generando un impacto directo en la noción de determinismo.

El proceso histórico que llevó a Boltzmann a presentar la interpretación estadística de la Segunda Ley de la termodinámica, y su posterior aceptación por parte de la comunidad científica, es rico en confrontaciones y desacuerdos. El periodo comprendido entre 1876 y 1895 fue un periodo controversial respecto del significado estadístico de la Segunda Ley, y tiene, en consecuencia, un profundo valor epistémico, pues nos muestra el proceso dinámico que supone la asimilación de novedades por parte de la comunidad científica; en este caso, la asimilación del estilo estadístico en la física. A grandes rasgos, las controversias con Boltzmann sobre la interpretación estadística empiezan en 1876 cuando Loschmidt señala algunos puntos problemáticos del trabajo de 1872 de Boltzmann donde este propone, por primera vez, el que hoy llamamos Teorema H. La crítica de Loschmidt, que hoy se conoce como "paradoja de Loschmidt", apunta a demostrar que es imposible derivar la Segunda Ley de la termodinámica exclusivamente a partir de los principios de la mecánica clásica. El interés central de Loschmidt en esta crítica es llamar la atención sobre la irreversibilidad, y el efecto que tuvo en la réplica de Boltzmann fue un fortalecimiento de su enfoque estadístico.

La discusión de Boltzmann con Loschmidt condujo a algunos intercambios que se publicaron en la revista Wiener Berichte (18761877), y fue a través de ellos que Boltzmann replanteó el enfoque de su explicación. Los enunciados genuinamente probabilistas para la explicación del equilibrio térmico empiezan a hacerse cada vez más evidentes:

Dado que hay infinitamente más distribuciones de estados uniformes que no uniformes, el último caso es extraordinariamente improbable y puede considerarse imposible a efectos prácticos; así como puede considerarse imposible que si uno comienza con oxígeno y nitrógeno mezclados en un recipiente, después de un mes encontrará oxígeno 
químicamente puro en la mitad inferior y nitrógeno en la mitad superior, pues aunque según la teoría de la probabilidad esto es sumamente improbable, no es completamente imposible. (Boltzmann 2003b [1877], p. 366; véase la discusión en Kuhn 1987 [1978]. La traducción es mía.)

Con este nuevo enfoque admitía que

un mismo valor de $f$ [función de distribución de velocidades] en $t_{0}$ es compatible con un número infinito de configuraciones de las moléculas dentro del intervalo inicial de velocidades, cada una de las cuales corresponde a un cierto microestado mecánico diferente. Por lo tanto, una única evolución temporal de la función $f$ "resume" evoluciones mecánicas completamente diferentes, y es aquí, precisamente, donde entra en juego el cálculo de probabilidades. (Lombardi 2009, p. 133)

El giro de Boltzmann respecto de sus ideas acerca de la Segunda Ley radica en que abandona el enfoque cinético con el que intentaba explicar la distribución de velocidades a partir de mecanismos de colisión entre las moléculas del gas, y adopta un enfoque mucho más general en el que recurre al valor de la probabilidad de una cierta distribución, independientemente de cómo esa configuración llega a darse. Aunque esta discusión giró explícitamente en torno al problema de la irreversibilidad, tuvo consecuencias directas en la interpretación estadística y por consiguiente en la concepción del determinismo.

Este es un punto fundamental para el núcleo de la controversia que nos ocupa, pues representa un giro drástico en la concepción de determinismo y su relación con las ideas de causalidad y ley física. La noción de ley, en su sentido mecánico más estricto, no puede albergar ninguna probabilidad, pues sería necesario aceptar la existencia de un azar subyacente en el comportamiento de la materia. $\mathrm{Y}$ recordemos que el azar, al ser incognoscible, fue descartado como posible constituyente del mundo. Así, la postulación del estado de un sistema no como necesario sino como probable implicó la reformulación del determinismo asociado a una concepción de ley como necesidad, así como la postulación de leyes que admitieran, en su seno, la probabilidad y el azar. Para este periodo, en el que se dan las controversias sobre la interpretación estadística de la Segunda Ley, ya algunos científicos habían dado el giro hacia la aceptación de la ley de distribución como genuina ley física, entre ellos Maxwell y Boltzmann principalmente; pero habría que esperar hasta 1895 para que esta asimilación empezara a hacerse más popular entre los expertos. 
En agosto de 1894, The British Association for the Advancement of Science realizó su congreso periódico en Oxford. En dicho encuentro, uno de los temas principales a debatir era la segunda parte del informe que, sobre el conocimiento de la termodinámica, dicha asociación le había encargado a George H. Bryan unos años antes. Como consecuencia de este encuentro y a partir de una carta enviada a Nature por Edward P. Culverwell (1894), en octubre de ese mismo año, se discutieron diferentes aspectos e implicaciones del teorema $\mathrm{H}$ de Boltzmann. Nuevamente, como en la discusión con Loschmidt, los aspectos centrales giraron en torno a la irreversibilidad; pero es importante resaltar aquí que esos intercambios permitieron a la comunidad científica profundizar sobre las consecuencias de la introducción de la probabilidad en las leyes de la física, y representan, en consecuencia, el comienzo de la aceptación de un determinismo estadístico, condición necesaria para el desbloqueo conceptual de la controversia.

Para finales del siglo XIX, el espacio controversial estaría configurado como se ilustra en la Figura 1 (p. 107). Allí pueden verse los diferentes elementos que constituyen el espacio, así como algunas de sus transformaciones: hay controversias, como el libre albedrío, que salen del foco e ingresan otras como la interpretación de la probabilidad.

El terreno común cambia aquí principalmente con respecto al siglo XVIII debido a la introducción de dos nuevas teorías: la termodinámica y el electromagnetismo, lo que implica el fortalecimiento del programa de reducción y unificación. Además, la aplicación de los métodos estadísticos al análisis de poblaciones, como ya hemos señalado, implicó una reformulación del concepto de ley, por fuera de la física. Para finales del siglo XIX ya se empieza a dar una aceptación de un determinismo estadístico como consecuencia de la aceptación de leyes estadísticas. Este nuevo determinismo se caracteriza porque, si bien se considera que el sistema contiene las variables físicas de estado bien determinadas, cierto tipo de fenómenos y los procesos que sufren en el tiempo no permiten conocer completamente toda la información del estado micro en términos mecánicos, sino estadísticos. Es aquí donde se hacen necesarios los nuevos objetos para explicar sistemas como un gas, en el que no es posible conocer el estado de cada una de las partículas.

La última década del siglo XIX y la primera del XX fueron el periodo de la asimilación generalizada de los métodos estadísticos. Aún así, para este periodo, podemos encontrar a Boltzmann intentando 


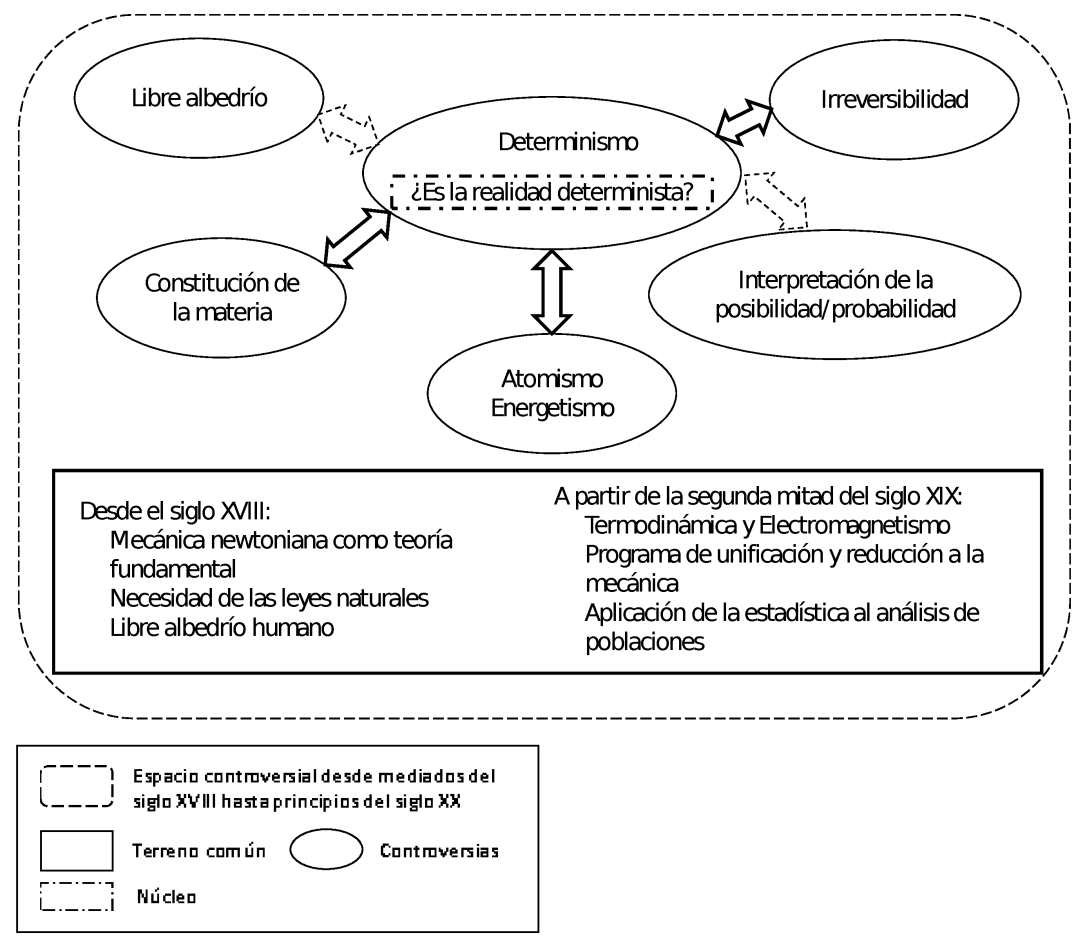

Figura 1: Estructura del espacio controversial.

explicar, una vez más, su nuevo enfoque probabilista. En una réplica que escribe a una crítica de Zermelo (nuevamente sobre el problema de la irreversibilidad) se queja de la poca recepción que han tenido sus ideas en Alemania y explica el énfasis probabilista de la Segunda Ley:

A menudo he enfatizado, lo más claramente posible, que la ley de distribución de Maxwell de velocidades entre las moléculas de gas no es en modo alguno un teorema de la mecánica ordinaria que se pueda probar solo con las ecuaciones de movimiento; por el contrario, solo se puede demostrar que tiene una probabilidad muy alta y que, para una gran cantidad de moléculas, todos los demás estados tienen, en comparación, una probabilidad tan pequeña que, a efectos prácticos, pueden ignorarse. Al mismo tiempo, también he enfatizado que la segunda ley de la termodinámica es, desde el punto de vista molecular, 
una ley meramente estadística. (Boltzmann 2003a [1896], p. 393; véase Stöltzner 1999. La traducción es mía.)

Vemos, entonces, cómo se relacionaron en este periodo las discusiones sobre irreversibilidad, probabilidad, mecánica estadística y determinismo. El hecho de que la noción de determinismo fuera aún difusa hacía que su relación con la idea de necesidad, ley física y causalidad levantara sospechas sobre los resultados probabilistas de Boltzmann. El indeterminismo epistémico asociado a estos métodos ingresa al foco de este espacio porque "si la mecánica estadística describe un sistema macroscópico especificado de un modo incompleto desde un punto de vista mecánico, las probabilidades deben ser interpretadas como probabilidades por ignorancia" (Lombardi 2009, p. 137). Si la necesidad es ley, la naturaleza deberá actuar de modo que cada estado sea posible o imposible, no meramente probable. Y esto abre un capítulo interesante sobre la interpretación de la probabilidad en términos de mera ignorancia o, como sucederá más adelante, con la interpretación de la ley como regularidad estadística, admitiendo así un indeterminismo ontológico.

Ahora bien, respecto de la relación entre determinismo y probabilidad, no está de más resaltar que una de las condiciones para la aplicación del determinismo en una teoría es la existencia de una relación unívoca entre el estado de un sistema y un instante temporal. Pero recordemos que, para la demostración del Teorema $\mathrm{H}$, Boltzmann propone que, para cada instante, un mismo valor de $f$ (función de distribución de velocidades) depende de un número infinito de configuraciones mecánicas. En este punto se evidencia la consolidación de la explicación estadística, pues se abandona la explicación de la distribución a partir de las causas (velocidades y colisiones moleculares individuales) y se adopta una genuina explicación estadística, intentando determinar las distribuciones más probables, independientemente de sus causas. Con la aceptación de este tipo de explicaciones, el estilo de razonamiento estadístico adquiere finalmente su autonomía.

\subsection{Gibbs: la consolidación de los métodos estadísticos y el desbloqueo conceptual}

El papel de las ideas de Gibbs fue determinante para cerrar algunos de los desacuerdos que hemos presentado hasta aquí. En el prefacio de su obra de 1902, Elementary Principles in Statistical Mechanics, Gibbs explica claramente que su interés no apunta a la resolución de 
los problemas de la termodinámica, sino a fundamentar una mecánica estadística como una teoría más general que facilite métodos y herramientas fundamentales que puedan aplicarse a diferentes problemas de la física. Por este motivo prescinde de cualquier hipótesis sobre la constitución de la materia y evita introducirse en las controversias sobre la reducción de la termodinámica a la mecánica, es decir, intenta mantenerse al margen de la controversia sobre la irreversibilidad. Al respecto dice en su obra:

Además, evitamos las dificultades más graves cuando, renunciando al intento de formular hipótesis sobre la constitución de los cuerpos materiales, realizamos investigaciones estadísticas como una rama de la mecánica racional. En el estado actual de la ciencia, parece casi imposible formular una teoría dinámica de la acción molecular que abarque los fenómenos de la termodinámica, la radiación y las manifestaciones eléctricas que acompañan la unión de los átomos. (Gibbs 1902, p. 13; la traducción es mía.)

El propósito de Gibbs es fundamentar una nueva física esencialmente estadística, y presentar de forma canónica sus ecuaciones y sus métodos propios. Así, se aleja de las controversias del viejo continente y da autonomía, de forma definitiva, a los métodos estadísticos para la física clásica. No obstante, como señala Brush (1976), es esta consolidación de los métodos estadísticos lo que abrirá definitivamente las puertas al indeterminismo ontológico inherente a la mecánica cuántica que aparecería unas décadas más tarde. Es decir, en el ámbito de la física la obra de Gibbs es el último paso necesario para el desbloqueo conceptual de la controversia.

Sin embargo, para la época en la que Gibbs publica su obra, en el campo de la filosofía ya algunos autores ponían en duda la idea de un determinismo ontológico. Peirce, por ejemplo, en su artículo "The Architecture of Theories" publicado en The Monist, plantea dudas sobre los tradicionales conceptos de ley física, causalidad y determinismo, al afirmar:

la única manera posible de dar cuenta de las leyes de la naturaleza y de la uniformidad en general es suponer que son resultado de la evolución. Esto supone que no son absolutas, que no son obedecidas con precisión. Supone un elemento de indeterminación, de espontaneidad o de azar absoluto en la naturaleza. Igual que, cuando tratamos de verificar cualquier ley física, encontramos que no puede satisfacer de forma precisa nuestras observaciones, y atribuimos correctamente la discrepancia 
a errores de observación, así tenemos que suponer la existencia de discrepancias mucho más diminutas debidas a la fuerza imperfecta de la ley misma, a una cierta desviación de los hechos respecto de cualquier fórmula definida. (Peirce 2012b [1891], p. 332)

En este párrafo convergen claramente los problemas del determinismo epistémico y ontológico, además de las nociones de causalidad y de ley física. Tanto en este artículo, como en "The Doctrine of Necessity Examined" (2012a [1892]), Peirce pone de manifiesto los primeros indicios de lo que Hacking (1990) llama la erosión del determinismo. En efecto, a las puertas del siglo XX, el acuerdo sobre el determinismo ontológico ya comienza a quebrarse, tanto en el ámbito científico como en el filosófico.

Finalmente, dada la relevancia que la hipótesis atómica tuvo en la movilización de las controversias de este espacio, no puede cerrarse esta primera etapa sin mencionar brevemente los eventos de 1905 respecto de la aceptación de la existencia de los átomos por parte de la comunidad científica. El artículo de Einstein (1905) titulado "Ueber die von der molekular-kinetischen Theorie der Wärme geforderte Bewegung von in ruhended Flussigkeiten suspenierten Teilchen" y las posteriores contrastaciones experimentales que Perrin realizó fueron determinantes para que la existencia de los átomos dejara de considerarse una hipótesis. Este es el argumento que brinda respaldo final al enfoque probabilista de Boltzmann. Con estas controversias y con los nuevos elementos conceptuales que hemos señalado aquí, el espacio controversial está listo para sacar a la luz la discusión sobre el determinismo ontológico. Por las profundas implicaciones que esta controversia tuvo para la física del siglo XX, reservo la presentación de ese periodo del espacio controversial en un estudio aparte.

\section{Conclusiones: indeterminismo epistémico en el marco de un determinismo ontológico}

En el presente trabajo he mostrado la estructura del espacio controversial acerca del determinismo desde mediados del siglo XIX hasta la primera década del siglo XX. Es a partir de este espacio así estructurado que se desarrollará la controversia en torno al determinismo ontológico asociada a la mecánica cuántica. Mi propósito fue reconstruir la red de controversias que aparecen en el espacio durante el periodo precontroversial, y mostrar cómo estas controversias iniciales llevaron a los actores al escepticismo respecto de un terreno común en el que el determinismo ontológico era aceptado por la comunidad 
científica de un modo generalizado. Partimos de algunos supuestos que ya señalaron Brush (1976), Hacking (1975, 1990) y Lombardi (2009) sobre la relación de los métodos estadísticos introducidos durante la segunda mitad del siglo XIX, la controversia sobre la irreversibilidad, y los cambios acaecidos alrededor del determinismo en la física hasta la primera década del siglo XX. También consideramos fundamental la hipótesis atomista que, si bien fue una controversia menos extensa que las demás (en este espacio en particular), resultó central para la introducción de la estadística en la física. Sobre la base de estos supuestos, y usando las herramientas conceptuales de los espacios controversiales y los estilos de razonamiento científico, logramos mostrar bajo una nueva luz cómo estos elementos están relacionados con las controversias de la red durante la primera etapa del espacio controversial.

Cerramos este primer estudio en el momento en el que todos los elementos conceptuales necesarios para el desbloqueo conceptual de la controversia sobre el determinismo han sido introducidos en la estructura del espacio: la aceptación de la mecánica estadística, a partir de los trabajos de Gibbs, y la aplicación de ésta a los problemas de la termodinámica, lo que representa la autentificación del estilo de razonamiento estadístico, además de la aceptación de la hipótesis atómica. En la segunda etapa de este espacio controversial estos acuerdos desembocarán en una discusión mucho más profunda que terminará por poner en duda la naturaleza determinista del mundo. Pero éste será el tema de un próximo artículo, continuación del presente trabajo. ${ }^{3}$

\section{BIBLIOGRAFÍA}

Álvarez, M., 2019, "Crisis de la física newtoniana y la primera etapa de la mecánica cuántica en la República de Weimar", en O. Nudler (ed.), El mundo amenazado: Las crisis globales y su repercusión en las ciencias,

${ }^{3}$ Este artículo se escribió en el marco de una estancia de investigación de movilidad internacional del doctorado en "Lógica y Filosofía de la Ciencia", financiada por la Universitat de València y llevada a cabo en la Universidad de Buenos Aires bajo la dirección de la Dra. Olimpia Lombardi, a quien quiero agradecer su dedicación y destreza en la estructuración de este trabajo, así como a su grupo por las discusiones y recomendaciones. A mi director, el Dr. Jesús Alcolea Banegas por sus diligentes correcciones y su incondicional apoyo. También, por su ayuda desinteresada, al Dr. Boris Ángelo Rodriguez (Universidad de Antioquia) por sus orientaciones en los aspectos técnicos de este trabajo. 
la filosofía y la literatura en el primer tercio del siglo XX, Editorial UNRN, Viedma, pp. 87-98.

Beauchamp, T., 1987, "Ethical Theory and the Problem of Closure", en Engelhardt and Caplan (eds.), Scientific Controversies: Case Studies in the Resolution and Closure of Disputes in Science and Technology, Cambridge University Press, Nueva York, NY.

Bishop, R.C., 2006, "Determinism and Indeterminism", en D.M. Borchert (ed.), Encyclopedia of Philosophy, vol. 3, Thompson Gale, Farmington Hills, MI.

Boltzmann, L., 2003a [1896], "Reply to Zermelo's Remarks on the Theory of Heat", en The Kinetic Theory of Gases, trad. S.G. Brush, Imperial College Press, Londres, pp. 392-402. Publicado originalmente en Annalen der Physik, vol. 193, no. 4, pp. 773-784.

, 2003b [1877], "On the Relation of a General Mechanical Theorem to the Second Law of Thermodynamics", trad. S.G. Brush, The Kinetic Theory of Gases, Imperial College Press, Londres, pp. 362-367. Publicado originalmente en Sitzungsberichte der kaiserlichen Akademie der Wissenschaften, part. II, 75, pp. 67-73.

Brush, S.G., 2003, The Kinetic Theory of Gases, Imperial College Press, Londres.

- 1983 , Statistical Physics and the Atomic Theory of Matter, from Boyle and Newton to Landau and Onsager, Princeton University Press, Princeton, NJ.

- 1976, The Kind of Motion We Call Heat: A History of the Kinetic Theory of Gases in the 19th Century, North-Holland Publishing Company, Amsterdam.

Butterfield, J., 1998, "Determinism and Indeterminism", en Edward Craig (ed.), Routledge Encyclopedia of Philosophy, vol. 3, Routledge, Londres, pp. 33-39.

Crombie, A.C., 1995, "Commitments and Styles of European Scientific Thinking", History of Science, vol. 33, no. 2, pp. 225-238.

Culverwell, E., 1894, “Dr. Watson's Proof of Boltzmann's Theorem on Permanence of Distributions", Nature, vol. 50, no. 617.

De Moivre, A., 1738, The Doctrine of Chances: Or, a Method of Calculating the Probability of Events in Play (ed. en línea 2014) (Cambridge Library Collection - Mathematics), Cambridge University Press, Cambridge.

Earman, J., 1986, A Primer on Determinism, D. Reidel Publishing Company, Dordrecht.

Einstein, A., 1905, “Ueber die von der molekular-kinetischen Theorie der Wärme geforderte Bewegung von in ruhended Flussigkeiten suspenierten Teilchen", Annalen der Physik, series 4, 17, pp. 549-560, en S.G. Brush, "A History of Random Processes. I. Brownian Movement from Brown to Perrin", Archive for History of Exact Sciencies, vol. 5, no. 1, 1968, pp. 1-36. 
Forman, P., 1984 [1971], Cultura en Weimar, causalidad y teoría cuántica: 1918-1927, trad. José Manuel Sanchez Ron, Alianza, Madrid. "Weimar Culture, Causality and Quantum Theory, 1918-1927", publicado originalmente en Historical Studies in the Physical Science, vol. 3, pp. 1-115.

Gibbs, J.W., 1902, Elementary Principles in Statistical Mechanics, Yale University Press, Cambridge.

Hacking, I., 1992a, "Style' for Historians and Philosophers", Studies in History and Philosophy of Science, vol. 23, no. 1, pp. 1-20.

—, 1992b, "Statistical Language, Statistical Truth, and Stadistical Reason: The Self-Authentication of a Style of Scientific Reasoning", The Social Dimensions of Science, en E. McMullin (ed.), University of Notre Dame Press, Notre Dame, pp. 130-157.

- 1990, The Taming of Chance, Cambridge University Press, Cambridge. [Versión al castellano: La domesticación del azar, trad. Alberto L. Bixio, Editorial Gedisa, Buenos Aires, 2012.]

, 1982, "Language, Truth and Reason", en Rationality and Relativism, Hollis and Lukes (eds.), The MIT press, Cambridge, Mass.

, 1975, The Emergence of Probability (reimpresión 2006), Cambridge University Press, Cambridge.

Hertz, H., 1899, "Author's Preface", en The Principles of Mechanics Presented in a New Form, trad. D.E. Jones y J.T. Walley, MacMillan and Co., Nueva York, NY.

Hume, D., 1902 [1748], Enquiries Concerning Human Understanding, en L.A. Selby-Bigge (ed.), Oxford University Press, Oxford.

— 1888 [1739], A Treatise of Human Nature, en L.A. Selby-Bigge (ed.), reimpresión 1967, Oxford University Press, Oxford.

James, W., 1897, The Will to Believe and Other Essays in Popular Philosophy, Dover Publications, New York. [Versión al castellano: La voluntad de creer y otros ensayos de filosofía popular, trad. Ramón Vilà Vernis, Marbot, Barcelona, 2009.]

Klein, M.J., 1973, "The Development of Boltzmann's Statistical Ideas", Acta Physica Austriaca, Suppl. X, pp. 53-106 Kragh, H., 2014, "A Sense of Crisis: Physics in the fin-de-siècle Era", en M. Saler (ed.), The Fin-de-Siècle World, Routledge, Nueva York.

Kuhn, T.S., 1987 [1978], La teoría del cuerpo negro y la discontinuidad cuántica, 1894-1912, trad. Paredes Larrueca, Alianza, Madrid.

Laplace, P.S., 1996 [1814], Ensayo filosófico sobre las posibilidades, trad. Pilar Castrillo, Altaya, Madrid. Publicado originalmente como Essai Philosophique sur les Probabilités, Courcier, París.

Lombardi, O., 2009, "El problema de la irreversibilidad, de Fourier a la teoría del caos", Espacios controversiales, en O. Nudler (ed.), Miño y Dávila, Buenos Aires, pp. 129-161.

— 2000, El problema del determinismo en la física, tesis doctoral, Facultad de Filosofía y Letras, Universidad de Buenos Aires, Buenos Aires. 
Mazur, A., 1981, The Dynamics of Technical Controversy, Communications Press, Washington, DC.

McMullin, E., 1987, "Scientific Controversy and its Termination", en Engelhardt and Caplan (eds.), Scientific Controversies: Case Studies in the Resolution and Closure of Disputes in Science and Technology, Cambridge University Press, Cambridge.

Nudler, O., 2009, Espacios controversiales. Hacia un modelo de cambio filosófico y científico, Miño y Dávila, Buenos Aires.

__ 2004, "Hacia un modelo de cambio conceptual: espacios controversiales y refocalización", Revista de Filosofía, vol. 29, no. 2, pp. 7-19.

—_, 2002, "Campos controversiales y progreso en filosofía. Manuscrito", Revista Internacional de Filosofía, vol. 25, no. 2, pp. 337-352.

Peirce, Ch.S., 2012a [1892], "La doctrina de la necesidad examinada", revisado en Obra filosófica reunida. Tomo I (1867-1893), en N. Houser y Ch. Kloesel (eds.), Fondo de Cultura Económica, Ciudad de México, pp. 344-356. "The Doctrine of Necessity Examined", publicado originalmente en The Monist, vol. 2, no. 3, abril, pp. 321-337.

__ 2012b [1891], "La arquitectura de las teorías", revisado en Obra filosófica reunida. Tomo I (1867-1893), en N. Houser y Ch. Kloesel (eds.), Fondo de Cultura Económica, Ciudad de México, pp. 331-343. "The Architecture of Theories", publicado originalmente en The Monist, vol. 1, no. 2, enero, pp. 161-176.

Stöltzner, M., 1999, "Vienna Indeterminism: Mach, Boltzmann, Exner", Synthese, vol. 119, no. 1/2, pp. 85-111.

Recibido el 17 de agosto de 2020; aceptado el 28 de agosto de 2020. 